\title{
EVIDENCE FOR A DRAMATIC ACTIVITY DECLINE IN THE NUCLEUS OF THE RADIO GALAXY FORNAX A
}

\author{
N. IYOMOTO, K. MAKISHIMA, M. TASHIRO, K. MATSUSHITA, \\ Y. FUKAZAWA, H. KANEDA AND S. OSONE \\ Department of Physics, University of Tokyo, \\ 7-3-1 Hongo, Bunkyo-ku, Tokyo 113, Japan
}

Fornax A (NGC 1316) is a radio galaxy with prototypical double lobes. Feigelson et al. (1995 ApJ 449, L149) and Kaneda et al. (1995 ApJ 454, L13) detected inverse Compton X-rays for the first time from its radio lobes, and unambiguously derived the lobe magnetic field intensity of 2-4 $\mu \mathrm{G}$. Accordingly, the radio-emitting electrons in the lobes are inferred to have a Lorentz factor of $10^{4}$, and hence a synchrotron life time of $\sim 10^{8} \mathrm{yr}$. This means that Fornax A was highly active at least $10^{8}$ years ago.

In contrast, the Fornax A nucleus is very faint in radio (Slee et al. 1994 MNRAS 269, 928), optical and UV (Fabbiano et al. 1994 ApJ 434, 67), although it remains uncertain whether the nucleus has switched off or is heavily absorbed. We re-analized the data obtained with ASCA on 1994 January 11 . The obtained spectra are dominated by thin thermal emission from hot inter-stellar medium, while the $2-10 \mathrm{keV}$ luminosity of hard excess, which may include AGN emission, is less than $1.2 \times 10^{40} \mathrm{erg} \mathrm{s}^{-1}$. This is at least three orders of magnitude lower than those of typical radio galaxy nuclei. Since fluorescence Fe-K line emission, a reliable index of absorption, is not statistically significant in ASCA spectra, X-ray emission from the Fornax A nucleus dose not suffer from heavy absorption; consequently, the nucleus of Fornax A is really inactive at present.

From these two results, we conclude that Fornax A has become dormant during the last $10^{8}$ years, due presumably to a dramatic decrease in the mass accretion rate. This conclusion suggests the possible abundance of "dormant" quasars in nearby galaxies. 